\title{
Relationship between Discrete Fourier Transformation and Eigenvalue Decomposition
}

\author{
Qun Wan, Li Hong Guo, Ding Wang, Lin Zou, and Ji Hao Yin
}

\begin{abstract}
Discrete Fourier transformation (DFT) of sample sequence and eigenvalue decomposition of sample correlation matrix are two of important tools and basic parts in signal and information processing. Since they are used to deal with the same random process, although from different viewpoint, there may be some intrinsic relationship between them. However, they are often introduced, explained and learned independently in the traditional textbooks and courses of signal and information processing. Here, we discuss some intrinsic relationship between the problems formulation of discrete Fourier transformation of sample sequence and eigenvalue decomposition of sample correlation matrix. The results of these lecture notes can help students deepen the understanding of their characteristics on simplicity, optimality and the reason why they are so popular and why we analyze and deal with signal and information processing by using discrete Fourier transformation of sample sequence and eigenvalue decomposition of sample correlation matrix.
\end{abstract}

Index Terms-Random process, autocorrelation matrix, Discrete Fourier transformation, eigenvalue decomposition.

\section{INTRODUCTION}

In the course of theory, algorithm and application of signal processing, Discrete Fourier Transformation (DFT) of sample sequence vector and eigenvalue decomposition of sample autocorrelation matrix are two popular tools and play an important role. Since they are used to analyze the same signal or random process, although from different viewpoint, there may be some intrinsic relationship between them. However, they are often introduced, explained and learned independently in the traditional textbooks and courses of signal and information processing. In addition, the students may wonder why $e^{j \omega t}$ is used in Discrete Fourier Transformation of sample sequence vector and why we use normalized vector in eigenvalue decomposition of sample autocorrelation matrix [1].

In this paper, we first introduce the intrinsic relationship behind the definition of Discrete Fourier Transformation of a stochastic process and eigenvalue decomposition of its autocorrelation matrix, from which the students can grasp

Manuscript received October 9, 2017; revised December 27, 2017. (Write the date on which you submitted your paper for review.) This work was supported by the project of fine course construction of master's degree (2016ZYXWJPKC002) and higher education personnel training quality and teaching reform (2016XJYYB006) in University of Electronic Science and Technology of China.

Qun Wan, Ding Wang, Lin Zou, and Ji hao Yin are with Dept. of Electric Engineering, University of Electrical Science and Technology of China, Chengdu, China (e-mail: wanqun@uestc.edu.cn).

Li Hong Guo is with Military Representative Agency in Jiujiang, Equipment Development Department, Central Military Commission. their related property and review the following knowledge points: the relationship between the variance of a random process and the power spectrum density function, and the relationship between power spectral density function of the input and output stochastic process of a linear time invariant filter [2].

Second, we discuss some intrinsic relationship between the problems formulation and introduced respectively the motivation to analyzing signal and information by using Discrete Fourier Transformation of sample sequence vector and eigenvalue decomposition of sample autocorrelation matrix [3]. It can provide a new perspective for students to understand why we analyze and deal with signal and information processing by using Discrete Fourier transformation of sample sequence and eigenvalue decomposition of sample correlation matrix.

Thirdly, in order to make the conclusions more rigorous, some derivations are provided. As the result, students could have a deeper understanding on the simplicity, optimality and the reason why Discrete Fourier Transformation of sample sequence vector and eigenvalue decomposition of sample autocorrelation matrix are so popular in the field of signal and information processing [4].

This lecture note is organized as follows. Relationship between the definition of Discrete Fourier transform of sample sequence vector and eigenvalue decomposition of sample autocorrelation matrix is introduced in the second section. Section III briefly formulates the problems associated with Discrete Fourier Transformation of sample sequence vector and eigenvalue decomposition of sample autocorrelation matrix. The next section presents the solution to the associated problems. The last section provides a concluding remark to summarize the lecture notes.

\section{RELATIONSHIP BETWEEN THE DEFINITION OF DISCRETE FOURIER TRANSFORMATION AND EIGENVALUE DECOMPOSITION}

\section{A. Definition}

In the course of signal and information processing, discrete Fourier transformation is usually used to estimate the power spectral density function which is defined as

$$
s(\omega)=\sum_{k=-\infty}^{+\infty} r(k) e^{-j \omega k}
$$

where

$$
r(k)=E\left(x(t) x^{*}(t-k)\right)
$$

is the autocorrelation function of random process $x(t)$. 
Given a sample sequence of random process, the power spectral density function estimated by DFT is given by

$$
\hat{s}(\omega, t)=\frac{1}{M}\left|\sum_{k=0}^{M-1} x_{k}(t) e^{-j \omega k}\right|^{2}=\mathbf{a}^{H}(\omega) \mathbf{x}(t)
$$

where

$$
\mathbf{x}(t)=\left[x_{0}(t) \quad x_{1}(t) \quad \mathrm{L} \quad x_{M-1}(t)\right]^{T}
$$

is a sample sequence vector,

$$
\mathbf{a}(\omega)=\frac{1}{\sqrt{M}}\left[\begin{array}{llll}
1 & e^{j \omega k} & \mathrm{~L} & e^{j(M-1) \omega}
\end{array}\right]^{T}
$$

On the other hand, eigenvalue decomposition of the sample correlation matrix of a random process is defined as

$$
\hat{\mathbf{R}}=\sum_{k=1}^{M} \lambda_{k} \mathbf{q}_{k} \mathbf{q}_{k}^{H}
$$

where $\lambda_{k}$ is the $k$-th eigenvalue in descending order, $\mathbf{q}_{k}$ is the corresponding eigenvector, and

$$
\hat{\mathbf{R}}=\sum_{t=1}^{N} \mathbf{x}(t) \mathbf{x}^{H}(t)
$$

is the sample correlation matrix.

Next, we will discuss the relationship between Discrete Fourier Transformation and eigenvalue decomposition of autocorrelation matrix, which is defined as

$$
\mathbf{R}=E\left(\mathbf{x}(t) \mathbf{x}^{H}(t)\right)
$$

\section{B. Relationship between Definitions}

As we know, there is a famous relationship between Discrete Fourier Transformation and eigenvalue decomposition of circular matrix. Circular matrix is defined as

$$
\mathbf{C}=\left[\begin{array}{cccc}
c_{0} & c_{1} & \mathrm{~L} & c_{M-1} \\
c_{M-1} & c_{0} & \mathrm{~L} & c_{M-2} \\
\mathrm{M} & \mathrm{M} & \mathrm{L} & \mathrm{M} \\
c_{1} & c_{2} & \mathrm{~L} & c_{0}
\end{array}\right\rfloor
$$

It is obvious that for $k=1,2, \ldots, M$, we have [5]

$$
\mathbf{C a}\left(\frac{k}{M}\right)=\lambda_{k} \mathbf{a}\left(\frac{k}{M}\right)
$$

where

$$
\lambda_{k}=\sum_{m=0}^{M-1} c_{k} e^{j \frac{k}{M} m}
$$

Therefore, vector given in (5) is eigenvector of circular matrix and the corresponding eigenvalue is Discrete Fourier Transformation of circular matrix elements.

Since Discrete Fourier Transformation and eigenvalue decomposition of autocorrelation matrix are used to describe the characteristics of the same random process, although from different viewpoint, there may be some intrinsic relationship between them. For example, we have

$$
S_{\min } \leq \lambda_{k} \leq S_{\max }
$$

where $\lambda_{k}$ is the eigenvalue of the autocorrelation matrix, $k=1,2, \ldots, M, S_{\min }$ and $S_{\max }$ is respectively the minimum and maximum value of the power spectral density of the same stationary random process.

Students who first get in touch with this inequality often have no way to start and feel that it is difficult to understand intuitively. However, from the point of view of signal processing, the derivation of the above inequality has a simple and clear signal processing significance.

First, the eigenvalue of the autocorrelation matrix is defined by [4]

$$
\lambda_{k}=\frac{\mathbf{q}^{H} \mathbf{R q}}{\mathbf{q}^{H} \mathbf{q}}
$$

where $\mathbf{R}$ and $\mathbf{q}$ is respectively the autocorrelation matrix and the eigenvector. The denominator is obviously nonnegative, and the nonnegative-definite property of autocorrelation matrix also shows the numerator is also nonnegative.

In fact, the numerator can be regarded as $r_{x}(0)$, i.e., the variance of a stochastic process $x(t)$, which is the output of a linear time invariant filter whose pulse response is given by $\mathbf{q}$, and the input is a stochastic process $u(n)$, whose autocorrelation matrix given as $\mathbf{R}$. Similarly, the denominator can also be regarded as $r_{y}(0)$, i.e., the variance of a stochastic process $y(t)$, which is the output of the same linear time invariant filter whose impulse response is given by $\mathbf{q}$, but the input is white noise process with unit variance. Therefore, we have

$$
\frac{\mathbf{q}^{H} \mathbf{R q}}{\mathbf{q}^{H} \mathbf{q}}=\frac{r_{x}(0)}{r_{y}(0)}
$$

Then, according to the relationship between the variance of a random process and the power spectrum density function [2], we have

$$
\frac{r_{x}(0)}{r_{y}(0)}=\frac{\int_{-\pi}^{\pi} S_{x}(\omega) d \omega}{\int_{-\pi}^{\pi} S_{y}(\omega) d \omega}
$$

where $S_{x}(\omega)$ and $S_{y}(\omega)$ is the power spectral density function of the random process $x(n)$ and $y(n)$, respectively.

Next, according to the relationship between power spectral density function of the input and output stochastic process of a linear time invariant filter, we have [2]

$$
\frac{\int_{-\pi}^{\pi} S_{x}(\omega) d \omega}{\int_{-\pi}^{\pi} S_{y}(\omega) d \omega}=\frac{\int_{-\pi}^{\pi} S_{u}(\omega) Q(\omega) d \omega}{\int_{-\pi}^{\pi} Q(\omega) d \omega}
$$


where $S_{u}(\omega)$ is he power spectral density function of the stochastic process $u(t), Q(\omega)$ is the amplitude square of the frequency response of the linear time invariant system whose impulse response is $\mathbf{q}$, i.e.,

$$
Q(\omega)=\left|\sum_{k=0}^{M-1} q_{k} e^{-j \omega k}\right|^{2}
$$

where

$$
\mathbf{q}=\left[\begin{array}{llll}
q_{0} & q_{1} & \mathrm{~L} & q_{M-1}
\end{array}\right]^{T}
$$

Thus we can directly obtain

$$
\lambda_{k}=\frac{\int_{-\pi}^{\pi} S_{u}(\omega) Q(\omega) d \omega}{\int_{-\pi}^{\pi} Q(\omega) d \omega} \leq \frac{\int_{-\pi}^{\pi} S_{\max } Q(\omega) d \omega}{\int_{-\pi}^{\pi} Q(\omega) d \omega}=S_{\max }
$$

and

$$
\lambda_{k}=\frac{\int_{-\pi}^{\pi} S_{u}(\omega) Q(\omega) d \omega}{\int_{-\pi}^{\pi} Q(\omega) d \omega} \geq \frac{\int_{-\pi}^{\pi} S_{\min } Q(\omega) d \omega}{\int_{-\pi}^{\pi} Q(\omega) d \omega}=S_{\min }
$$

In this section, we introduce the intrinsic relationship behind the definition of Discrete Fourier Transformation of a stochastic process and eigenvalue decomposition of its autocorrelation matrix, from which the students can grasp their related property and review the following knowledge points: (1) the relationship between the variance of a random process and the power spectrum density function. (2) The relationship between power spectral density function of the input and output stochastic process of a linear time invariant filter.

\section{RELATIONSHIP BETWEEN THE PROBLEMS FORMULATION}

\section{A. Problem Formulation of DFT}

Let us approximate a sample sequence vector $\mathbf{x}(t)$ by using vector $\mathbf{a}(\omega)$. This problem can be formulated as follows

$$
\min _{\substack{s(\omega, t) \\ t=1,2, \ldots, N}} \sum_{t=1}^{N}\|\mathbf{x}(t)-s(\omega, t) \mathbf{a}(\omega)\|_{F}^{2}
$$

The students may wonder why we use $\mathbf{a}(\omega)$ to approximate a sample sequence vector. The reason is that

$$
v(t)=e^{j \omega t}
$$

is 1 -dimensional signal, i.e., $v(t)=e^{j \omega} v(t-1)$. In other word, we want to describe or analyze a stochastic process by the simplest signal. It can be regarded as a motivation of Discrete Fourier Transformation.

\section{B. Problem Formulation of Eigenvalue Decomposition of Sample Correlation Matrix}

Similarly, let us approximate a sample sequence vector $\mathbf{x}(t)$ by using a normalized vector $\mathbf{q}$, which is independent of time. This problem can be formulated as follows

$$
\begin{gathered}
\min _{\substack{\mathbf{q}, \alpha(t) \\
t=1,2, \ldots, N}} \sum_{t=1}^{N}\|\mathbf{x}(t)-\alpha(t) \mathbf{q}\|_{F}^{2} \\
\text { s.t. } \quad \mathbf{q}^{H} \mathbf{q}=1
\end{gathered}
$$

Also, the students may wonder why we use a normalized vector $\mathbf{q}$ to approximate a sample sequence vector. The reason is that $\mathbf{q}$ represents 1-dimensional subspace in the space where sample sequence vector $\mathbf{x}(t)$ locates. In other word, we want to describe or analyze a stochastic process in the lowest dimensional subspace. It can be regarded as one of motivations of eigenvalue decomposition of sample autocorrelation matrix.

\section{RELATIONSHIP BETWEEN THE SOLUTIONS}

\section{A. Solution to Problem (21)}

It is easy to see that the solution to least squares problem (21) is

$$
\hat{s}(\omega, t)=\mathbf{a}^{H}(\omega) \mathbf{x}(t)=\frac{1}{\sqrt{M}} \sum_{k=0}^{M-1} x_{k}(t) e^{-j \omega k}
$$

for $t=1,2, \ldots, N$

\section{B. Solution to Problem (23)}

It is also easy to see that the optimal solution to problem (23) is

$$
\hat{\alpha}(t)=\mathbf{q}^{H} \mathbf{x}(t)
$$

Substituting it into (23)yield

$$
\begin{gathered}
\min _{\mathbf{q}} \sum_{t=1}^{N}\left\|\mathbf{x}(t)-\mathbf{q} \mathbf{q}^{H} \mathbf{x}(t)\right\|_{F}^{2} \\
\text { s.t. } \quad \mathbf{q}^{H} \mathbf{q}=1
\end{gathered}
$$

It is equivalent to

$$
\begin{gathered}
\min _{\mathbf{q}} \operatorname{Tr}\left(\left(\mathbf{I}-\mathbf{q} \mathbf{q}^{H}\right) \hat{\mathbf{R}}\left(\mathbf{I}-\mathbf{q q}^{H}\right)\right) \\
\text { s.t. } \quad \mathbf{q}^{H} \mathbf{q}=1
\end{gathered}
$$

where $\operatorname{Tr}$ is the trace of a matrix and $\hat{\mathbf{R}}$ is sample correlation matrix. Since we have

$$
\begin{aligned}
& \operatorname{Tr}\left(\left(\mathbf{I}-\mathbf{q q}{ }^{H}\right) \hat{\mathbf{R}}\left(\mathbf{I}-\mathbf{q q}{ }^{H}\right)\right) \\
& =\operatorname{Tr}\left(\hat{\mathbf{R}}\left(\mathbf{I}-\mathbf{q} \mathbf{q}^{H}\right)\left(\mathbf{I}-\mathbf{q q} \mathbf{q}^{H}\right)\right) \\
& =\operatorname{Tr}\left(\hat{\mathbf{R}}\left(\mathbf{I}-\mathbf{q q} \mathbf{q}^{H}\right)\right) \\
& =\operatorname{Tr}\left(\hat{\mathbf{R}}-\hat{\mathbf{R}} \mathbf{q q}{ }^{H}\right) \\
& =\operatorname{Tr}(\hat{\mathbf{R}})-\mathbf{q}^{H} \hat{\mathbf{R}} \mathbf{q}
\end{aligned}
$$

problem (27) is straightforwardly reduced to

$$
\begin{aligned}
& \underset{\mathbf{q}}{\max } \mathbf{q}^{H} \hat{\mathbf{R}} \mathbf{q} \\
& \text { s.t. } \quad \mathbf{q}^{H} \mathbf{q}=1
\end{aligned}
$$


Therefore, the solution is the eigenvector corresponding to the maximum eigenvalue of sample correlation matrix $\hat{\mathbf{R}}$, i.e.,

$$
\hat{\mathbf{R}} \mathbf{q}=\lambda_{\max } \mathbf{q}^{H}
$$

Substituting the above equation into (27), we attain the minimum value as

$$
\min _{\substack{\mathbf{q}, \alpha(t) \\ t=1,2, \ldots, N}} \sum_{t=1}^{N}\|\mathbf{x}(t)-\alpha(t) \mathbf{q}\|_{F}^{2}=\operatorname{Tr}(\hat{\mathbf{R}})-\lambda_{\max }=\sum_{k=2}^{M} \lambda_{k}
$$

In this section, we introduced respectively the motivation to analyzing signal and information by using Discrete Fourier Transformation of sample sequence vector and eigenvalue decomposition of sample autocorrelation matrix. In order to make the conclusions more rigorous, some derivations are provided. As the result, students could have a deeper understanding on their simplicity, optimality and the reason why Discrete Fourier Transformation of sample sequence vector and eigenvalue decomposition of sample autocorrelation matrix are so popular in the field of signal and information processing.

\section{CONCLUSION}

When we analyze a signal or random process by using 1-dimensional signal, we encounter the Discrete Fourier transformation of sample sequence, whereas by using 1-dimensional subspace, we encounter eigenvalue decomposition of sample correlation matrix. Though they are two of important tools dealing with the signal or random process from different viewpoint, there are some intrinsic relationship between them. Therefore, they should not be introduced, explained or learned independently in the course of signal and information processing. The intrinsic relationship between discrete Fourier transformation of sample sequence and eigenvalue decomposition of sample correlation matrix includes the definition, problems formulation and their solutions. The results of these lecture notes can help students deepen the understanding of their characteristics on simplicity, optimality and the reason why they are so popular and why we analyze and deal with signal and information processing by using discrete Fourier transformation of sample sequence and eigenvalue decomposition of sample correlation matrix.

\section{REFERENCES}

[1] Q. Wan, Y. Liu, L. Zou, and J. H. Yin, "Lecture notes on signal processing in high dimensional space," IEEE International Conference on Teaching, Assessment and Learning for Engineering (TALE'2015), Zhuhai, China, Dec 10-12, 2015, pp. 127-130.

[2] P. Stoica and R. Moses, Spectral Analysis of Signals, Prentice Hall, 2005

[3] Q. Wan, "Learn discrete fourier transform from the viewpoint of optimization in signal processing," in Proc. International Conference on Creative Education (ICCE 2013), Singapore, Sep. 21-23, 2013, pp. 89-92.

[4] Q. Wan, B. G. Xu, Y. H. Wan, S. L. Tang, X. K. Ding, and Y.L. Liu, "Unit-sparse data and covariance model fitting: Discrete fourier transformation and minimum variance distortless response," in Proc. 6th International Conference of Education, Research and Innovation, Seville, Nov. 18th-20th, 2013, pp. 5017-5021.

[5] J. D. Philip, Circulant Matrices, Wiley, New York, 1970.

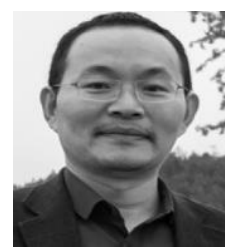

Qun Wan was born in March 1971. He received the B.Sc. degree in electronic engineering from Nanjing University, Nanjing, China, in 1993, the M.Sc. and $\mathrm{Ph} . \mathrm{D}$. degrees in electronic engineering from the University of Electronic Science and Technology of China (UESTC), Chengdu, China, in 1996 and 2001, respectively. From 2001 to 2003, hewas a Postdoctoral Researcher with the Department of Electronic Engineering, Tsinghua University. Since 2004, he has been a professor with the Department of Electronic Engineering, UESTC. He is currently the director of the Joint Research Lab of Array Signal Processing and the associate dean of School of Electronic Engineering. His research interests include direction finding, radio localization, and signal processing based on information criterion.

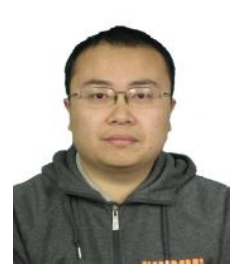

Lin Zou was born in Chengdu, in March 1982. He received the B.Sc. degree in electronic and information engineering from the University of Electronic Science and Technology of China (UESTC), Chengdu, China, in 2004, the M.Sc. and Ph.D. degrees in signal and information processing from the University of Electronic Science and Technology of China (UESTC), Chengdu, China, in 2007 and 2012, respectively. From 2010 to 2011, he was a joint-training Ph.D. with the Signal Processing Group, Department of Engineering, University of Cambridge, Cambridge, UK. He is an associate professor with the Department of Electronic Engineering, UESTC. His research interests include array signal processing, engineering applications of geometric algebra and hyper complex.

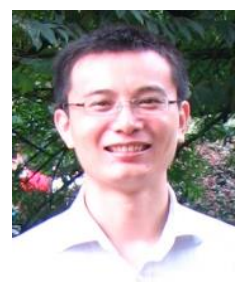

Jihao Yin received the Ph.D. degree in electrical engineering from University of Electronic Science and Technology of China, Chengdu, Sichuan, China in 2007. He is currently an associate professor in statistical signal processing with School of Information and Communication Engineering, University of Electronic Science and Technology of China. 